\title{
Numerical Analysis of a Dielectric Rod Antenna- Demonstration of the Discontinuity-Radiation Concept
}

\author{
Takashi Ando, Student Member, IEEE, Junji Yamauchi, Member, IEEE, and Hisamatsu Nakano, Fellow, IEEE
}

\begin{abstract}
A technique combining the finite-difference time-domain method and the imaginary-distance beam-propagation method is used to demonstrate the discontinuity-radiation concept for a dielectric rod antenna. The field near the rod is decomposed into a guided and an unguided wave. Using the two waves, the feed and terminal patterns are evaluated. It is numerically revealed that superposing the two patterns forms the radiation field of the antenna. The decomposition technique enables us to efficiently evaluate the directivity of a long rod antenna.
\end{abstract}

Index Terms-Dielectric antennas, dielectric waveguides, endfire antennas, finite-difference time-domain (FDTD) methods, finite difference methods.

\section{INTRODUCTION}

A DIELECTRIC rod antenna has been used as an endfire radiator for many years [1]-[10]. Experimental studies have been conducted at microwave and millimeter-wave frequencies [5]-[10]. Despite the extensive use of the antenna, no exact design procedure exists for them [10]. Theoretical methods usually involve simplifications and only provide general design guidelines [1]-[4].

The radiation mechanism of the dielectric rod antenna can be explained by the so-called discontinuity-radiation concept [1], in which the antenna is regarded as an array composed of two effective sources at the feed and free ends of the rod. Part of the power excited at the feed end is converted into guided-wave power and is transformed into radiation power at the free end. The remaining power is converted into unguided-wave power radiating near the feed end. Therefore, the directivity of the dielectric rod antenna is characterized by the directivities generated by the two effective sources. However, no exact calculation based on the discontinuity-radiation concept has been made for a practical rod antenna. This is due to the difficulty in quantitatively evaluating the radiation fields generated from the discontinuities at the feed and free ends.

On the other hand, a dielectric rod has received much attention in the waveguide analysis as well as the antenna analysis, since the knowledge of the eigenmode is of fundamental importance in the design of a dielectric waveguide circuit. Many methods have been proposed for this important issue [11]-[16]. Note, that the methods developed in [14]-[16] are based on Yee's mesh. The use of Yee's mesh has the advantage that the

Manuscript received January 11, 2001; revised August 20, 2002.

The authors are with the Faculty of Engineering, Hosei University, Koganei,

Tokyo 184-8584, Japan (e-mail: taka.ando@nifty.com; j.yma@k.hosei.ac.jp).

Digital Object Identifier 10.1109/TAP.2003.814731 obtained eigenmode fields can directly be utilized for the finite-difference time-domain (FDTD) method.

Recently, a technique combining the FDTD method and the eigenmode solver based on Yee's mesh [16], [17] has been proposed by the authors [18]. This technique enables us to decompose the total field near the rod into the guided and unguided waves. The technique can lead to deeper insight into the radiation mechanism of the dielectric rod antenna.

The purpose of this paper is to demonstrate the discontinuityradiation concept for a dielectric rod antenna fed by a metallic waveguide with a planar ground plane, and to show how the use of the discontinuity-radiation concept enhances the computational efficiency for the design of a long dielectric rod antenna.

The field near the rod is decomposed into the guided and unguided waves. Using the obtained waves, we can evaluate a feed pattern corresponding to the directivity generated by the effective source at the feed end and a terminal pattern corresponding to that at the free end. It is numerically revealed that superposing the feed and terminal patterns forms the radiation pattern of the dielectric rod antenna. The gain of a long rod antenna is efficiently calculated by superposing the feed and terminal patterns. The radiation characteristics calculated on the basis of the discontinuity-radiation concept agree well with experimental results.

\section{NUMERICAL METHODS}

\section{A. FDTD Analysis}

Fig. 1 shows the configuration of a dielectric rod antenna fed by a rectangular waveguide with a planar ground plane. The relative permittivity of the rod is chosen to be $\epsilon_{r, \text { rod }}=2.1$ (Teflon) [note, that some data for $\epsilon_{r, \text { rod }}=2.8$ (polycarbonate) will also be presented for comparison]. It is assumed that the metallic waveguide and the ground plane are perfectly conducting, and that the dielectric rod is lossless medium.

The total field in the computational region (see Fig. 2) is evaluated by the use of the FDTD method. The grid widths are taken to be $\Delta x=a / 22(\approx 1.04 \mathrm{~mm}), \Delta y=b / 10(=1.02 \mathrm{~mm})$, and $\Delta z=1 \mathrm{~mm}$. Owing to the symmetry of the configuration with respect to the $x-z$ and $y-z$ planes, the antenna system is analyzed using a quarter region of a full computational region: $\left(L_{x} / 2, L_{y} / 2, L_{z}\right)=(90 \Delta x, 90 \Delta y, 300 \Delta z)$.

The metallic waveguide is excited with the $\mathrm{TE}_{10}$ mode. For a continuous wave simulation of the $\mathrm{TE}_{10}$ mode, we use an excitation scheme of a $+z$-propagating incident waveform [19], [20]. 


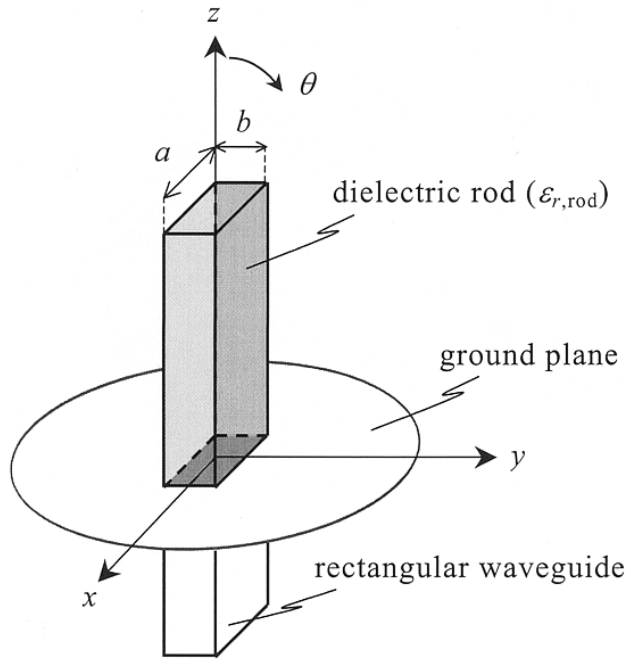

(a)

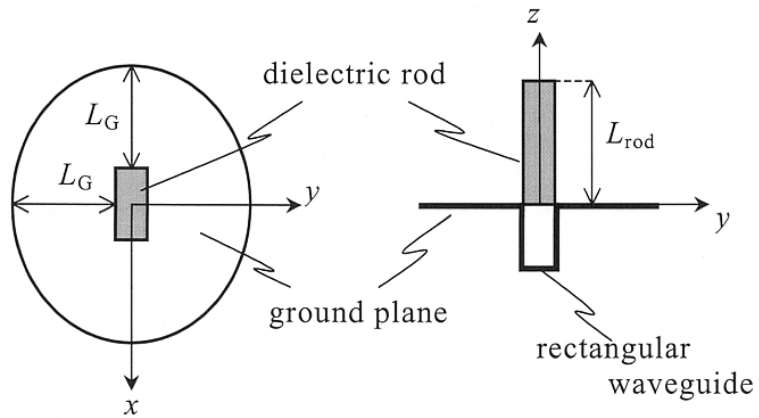

(b)

(c)

Fig. 1. Configuration of a dielectric rod antenna $\left[\epsilon_{r, \operatorname{rod}}=2.1, a=22.9 \mathrm{~mm}\right.$, $\left.b=10.2 \mathrm{~mm}, L_{\mathrm{G}}=\lambda_{0}, f=9 \mathrm{GHz}\left(\lambda_{0} \approx 33.3 \mathrm{~mm}\right)\right]$. (a) Overall geometry. (b) Top view. (c) Side view.

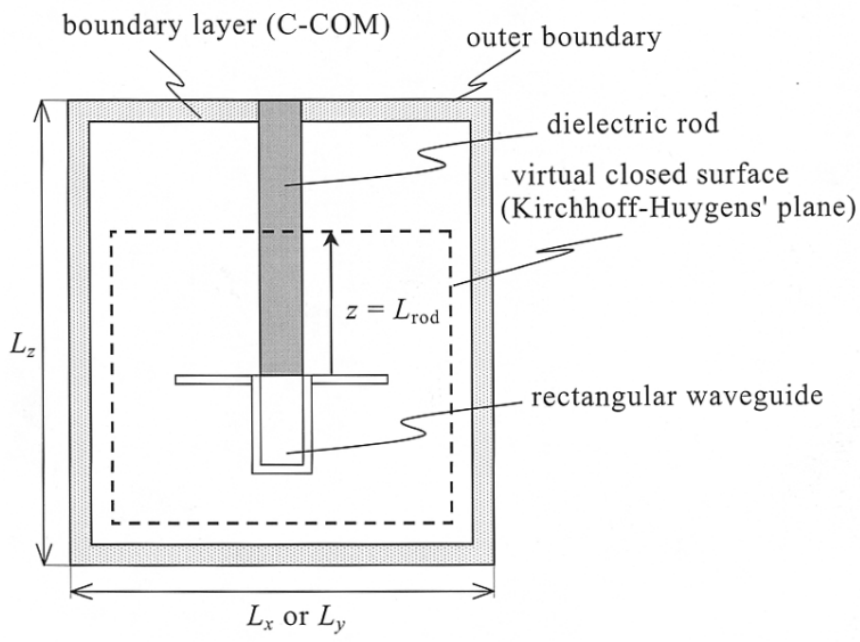

Fig. 2. Computational region.

The concurrent complementary operators method (C-COM) employing a second-order Higdon operator [21] is applied to the outer boundary of the computational region, as shown in Fig. 2. The C-COM can achieve a wide dynamic range in spite of the fact that only a few cells are required in the absorbing boundary region. In this analysis, four cells are allocated for the absorbing region.

\section{B. Eigenmode Analysis}

To decompose the total field near the rod into the guided and unguided waves, we first have to evaluate the field profile and the propagation constant $\beta_{0}$ of the eigenmode in the rod. For the eigenmode analysis, the use of Yee's mesh is desirable [14]-[16], since the obtained eigenmode field distributions can be naturally incorporated into the FDTD analysis. In this paper, the imaginary-distance beam-propagation method based on Yee's mesh (YM-BPM) [16], [17] is adopted for the analysis of the eigenmode in the rod.

The formulation of the YM-BPM begins with Maxwell's equations. We express the electric and magnetic fields as $\mathbf{E}(x, y, z, t)=\mathcal{E}(x, y, z) \exp \left[j\left(\omega t-\beta_{\text {ref }} z\right)\right]$ and $\mathbf{H}(x, y, z, t)=\mathcal{H}(x, y, z) \exp \left[j\left(\omega t-\beta_{\text {ref }} z\right)\right]$, where $\mathcal{E}(x, y, z)$ and $\mathcal{H}(x, y, z)$ are the slow-varying envelope functions of $z$, and $\beta_{\text {ref }}$ is the reference phase constant representing the fast-varying spatial phase. Following Lee's procedure [16], we obtain six difference equations for the field components based on Yee's mesh. It should be noted that $\mathrm{E}_{z}$ and $\mathrm{H}_{z}$ components are determined using the relations of $\nabla \cdot \epsilon \mathbf{E}=0$ and $\nabla \cdot \mathbf{H}=0$. As found in [16], the use of the divergence relations contributes to a reduction in round-off errors with subsequent stability of the numerical results.

We recall that the forward-traveling modes of a uniform waveguide along the $z$ direction can be expressed by a sum of normal modes

$$
\phi(x, y, z)=\sum_{m=0}^{\infty} a_{m} \mathrm{e}^{-j \beta_{m} z} \phi_{m}(x, y)
$$

where $\phi$ is any field component, $\phi_{m}$ is the eigenvector, $a_{m}$ is the amplitude for the forward-traveling mode, and $\beta_{m}$ is the propagation constant of the eigenmode. The subscript $m$ indicates the mode order.

$\phi$ is also written as

$$
\phi(x, y, z)=\varphi(x, y, z) \mathrm{e}^{-j \beta_{\text {ref }} z}
$$

where $\varphi$ is the slow-varying envelope function of $z$. Substituting (2) into (1), we obtain

$$
\varphi(x, y, z)=\sum_{m=0}^{\infty} a_{m} \mathrm{e}^{-j\left(\beta_{m}-\beta_{\mathrm{ref}}\right) z} \phi_{m}(x, y)
$$

We now change the coordinate $z$ in the propagation direction to $j \tau$, replacing the sinusoidal phase variation in the real axis $z$ propagation with the exponential amplitude variation in the imaginary axis $\tau$ propagation. Therefore, as an arbitrary input field propagates in the $\tau$ direction, the fundamental mode with the largest $\beta_{0}$ grows faster (decays slower) than the higher order modes for $\beta_{0}>\beta_{\text {ref }}\left(\beta_{0}<\beta_{\text {ref }}\right)$. In other words, an arbitrary input field that contains the lowest mode field converts into the lowest eigenmode field, as the input field propagates in the $+\tau$ direction. The field distributions of all components are calculated stepwise in the $+\tau$ direction. 


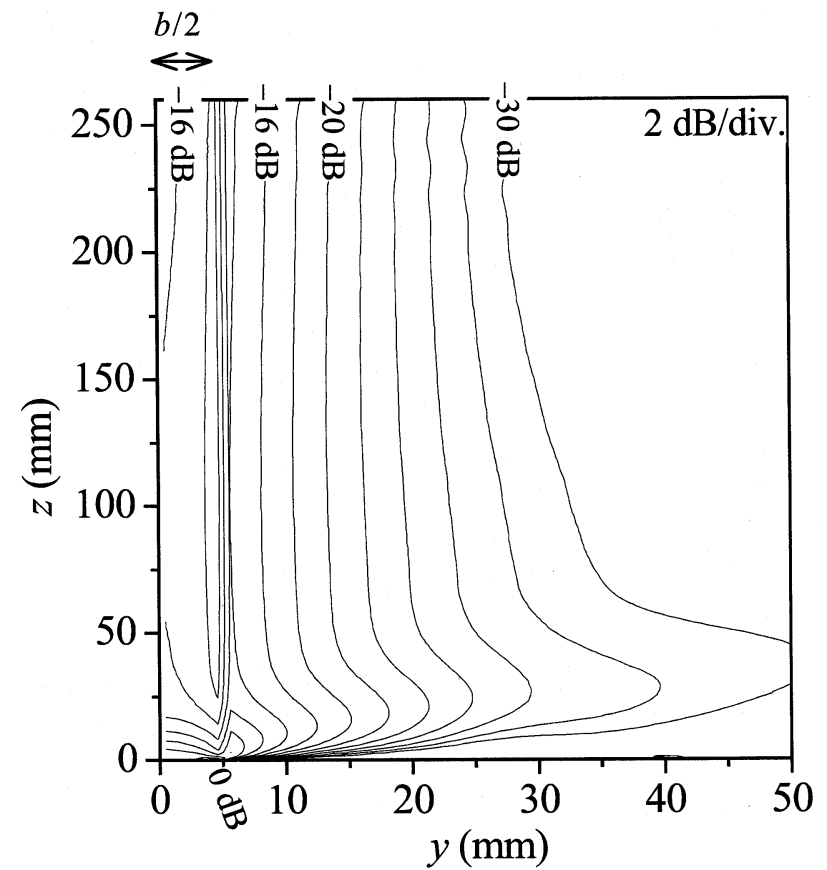

Fig. 3. Field distribution of $\mathrm{E}_{y}$ (half region).

After the convergence to the eigenmode field, $\beta_{0}$ is evaluated by the growth or decay in the propagating field amplitude of any component at $\tau$ and $\tau+\Delta \tau$

$$
\begin{aligned}
& \beta(\tau)=\beta_{\mathrm{ref}}+\lim _{\tau \rightarrow \infty} \\
& \times \frac{\iint\left[\ln \left\{\phi_{(x, y, \tau+\Delta \tau)}\right\}-\ln \left\{\phi_{(x, y, \tau)}\right\}\right]\left|\phi_{(x, y, \tau)}\right|^{2} d x d y}{\Delta \tau \iint\left|\phi_{(x, y, \tau)}\right|^{2} d x d y} \equiv \beta_{0} .
\end{aligned}
$$

In this paper, $\beta_{0}$ is obtained at a propagation distance of $\tau \approx$ $350 \mathrm{~mm}(=500 \Delta \tau)$, where the convergence of $\beta_{0}$ in six decimal places is obtained. Preliminary calculation shows that the dielectric rod treated in this paper operates as a single mode waveguide at testing frequencies. The cutoff frequency of the $\mathrm{E}_{21}^{y}$ mode for a rod of $\epsilon_{r, \text { rod }}=2.1$ is $12.2 \mathrm{GHz}$, and that for $\epsilon_{r, \text { rod }}=2.8$ is $9.9 \mathrm{GHz}$.

\section{Demonstration of THE DiscontinutTy-Radiation CONCEPT}

\section{A. Decomposition of Field}

Consideration is first given to the behavior of the guided and unguided waves generated from the discontinuity at the feed end. To investigate the essential properties of the two waves, we consider a case in which the reflected wave from the free end is eliminated. For this purpose, the free end is directly terminated with the condition based on the C-COM, as depicted in Fig. 2.

Fig. 3 shows the typical intensity distribution of $\mathrm{E}_{y}$ in the $y-z$ plane for $\epsilon_{r, \text { rod }}=2.1$. Since the field profile of the $\mathrm{TE}_{10}$ mode is different from that of the $\mathrm{E}_{11}^{y}$ mode in the dielectric rod [11], part of the power excited at the feed end is converted into the guided-wave power, and the remaining power is into the unguided-wave power. To interpret the behavior of the two

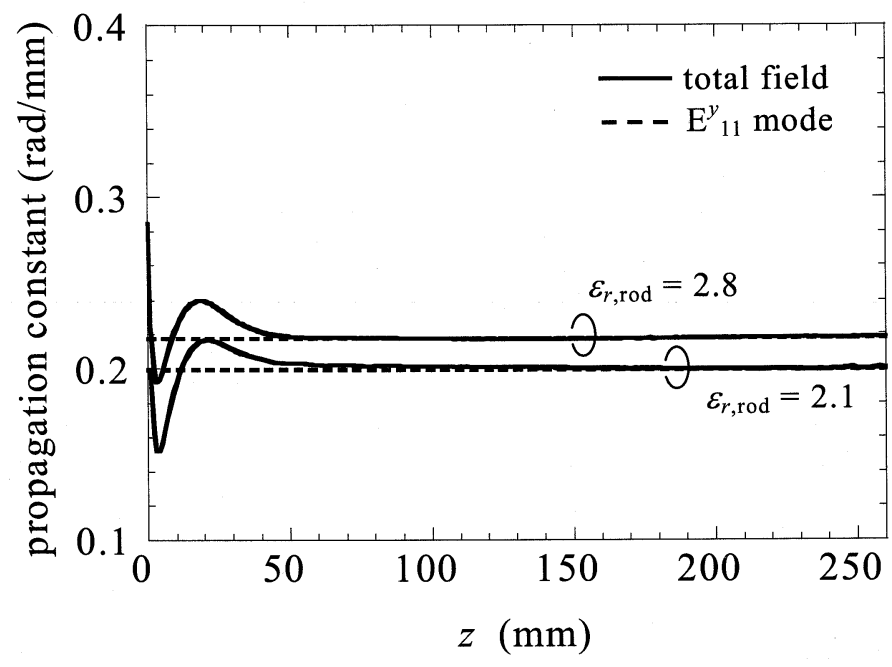

Fig. 4. Variation in propagation constant of $\mathrm{E}_{y}$.

waves, we decompose the total field near the rod, $\phi_{\text {total }}$, into the guided wave $\phi_{\mathrm{g}}$ and the unguided wave $\phi_{\mathrm{ug}}$

$$
\phi_{\text {total }}(x, y, z)=\phi_{\mathrm{g}}(x, y, z)+\phi_{\mathrm{ug}}(x, y, z) .
$$

Separating the guided wave into an amplitude term and a phase term, we write $\phi_{\mathrm{g}}$ as

$$
\phi_{\mathrm{g}}(x, y, z)=A_{\mathrm{e}} \phi_{\mathrm{eig}}(x, y) \exp \left[-j\left(\beta_{0} z-\psi_{0}\right)\right]
$$

where $\phi_{\text {eig }}(x, y)$ is the field profile of the eigenmode propagating along the rod, $A_{\mathrm{e}}$ is a coefficient of the amplitude, and $\psi_{0}$ is an initial phase of the surface wave at the feed end. $\phi_{\text {eig }}(x, y)$ and $\beta_{0}$ are calculated by the YM-BPM. To decompose the total field, we have to know $A_{\mathrm{e}}$ and $\psi_{0}$.

At sufficiently long distance $z=L_{\mathrm{r}}$ where the unguided-wave power is nearly eliminated, $\phi_{\text {total }}\left(x, y, z=L_{\mathrm{r}}\right)$ can be regarded as $\phi_{\mathrm{g}}\left(x, y, z=L_{\mathrm{r}}\right)$. In other words, the phase of $\phi_{\text {total }}\left(x, y, z=L_{\mathrm{r}}\right)$ almost becomes a reference phase $\psi_{\mathrm{r}}\left(=\beta_{0} L_{\mathrm{r}}-\psi_{0}\right)$ of the guided wave $\phi_{\mathrm{g}}\left(x, y, z=L_{\mathrm{r}}\right)$. To demonstrate this fact, the variation of the propagation constant as a function of $z$ is shown in Fig. 4. The propagation constant is calculated by evaluating the difference in the phase of the total field of $\mathrm{E}_{y}$ on the axis between two adjacent axial steps. We can find some fluctuation near the feed end, owing to the effects of the evanescent field, but the propagation constant rapidly converges to the eigenvalue as the field propagates. Note that $A_{\mathrm{e}}$ may also be determined from the amplitude of $\phi_{\text {total }}\left(x, y, z=L_{\mathrm{r}}\right)$. To increase the accuracy, however, we employ the least-squares technique described in [19], in which the orthogonality relation between the guided and unguided waves is utilized.

Once $\psi_{\mathrm{r}}$ is determined, $\psi_{0}$ is evaluated by $\psi_{0}=\beta_{0} L_{\mathrm{r}}-\psi_{\mathrm{r}}$. Consequently, $\phi_{\mathrm{g}}(x, y, z)$ in (6) is obtained from the determined $A_{\mathrm{e}}$ and $\psi_{0}$. Finally, $\phi_{\mathrm{ug}}(x, y, z)$ is calculated by subtracting $\phi_{\mathrm{g}}(x, y, z)$ from $\phi_{\text {total }}(x, y, z)$.

Fig. 5(a) and (b) illustrate the intensity distributions of the guided and unguided waves of $\mathrm{E}_{y}$ in the $y-z$ plane, respectively. The ratio of the total radiation power to the guided-wave power shows that the guided wave is excited with an efficiency of approximately $49 \%$ at the feed end. Since the decomposition technique separates the total field into the guided and unguided 


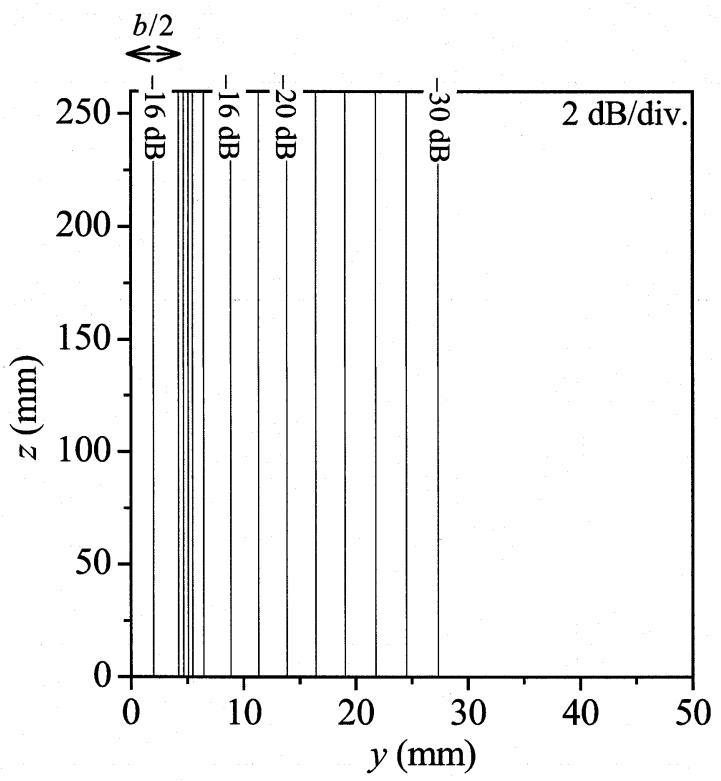

(a)

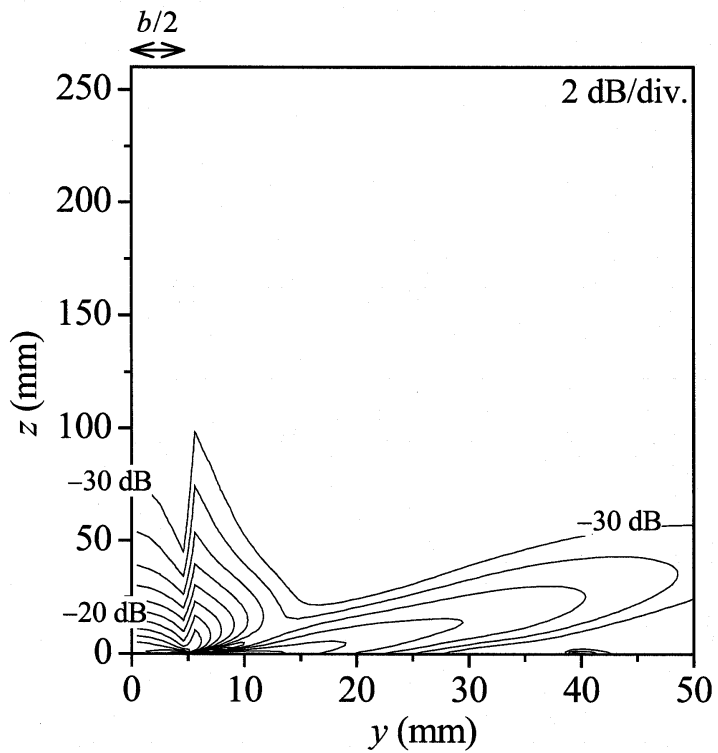

(b)

Fig. 5. Field distributions of $\mathrm{E}_{y}$ (half region). (a) Guided wave. (b) Unguided wave.

waves, we can separately evaluate the directivities generated by the two waves as will be discussed in Section III-B. It should be noted that the unguided wave is characterized by a leaky wave, which travels some distances along and close to the rod axis, and a space wave, which rapidly propagates away from the rod [22]. The existence of the leaky wave leads to the fact that, in the discontinuity-radiation concept, the feed source cannot be localized in contrast to the terminal source. We will, therefore, determine a sufficient length $L_{\mathrm{r}}$ to validate the present method in Fig. 7.

\section{B. Directivity}

Before the demonstration of the discontinuity-radiation concept, the directivity of the rod antenna is evaluated as a

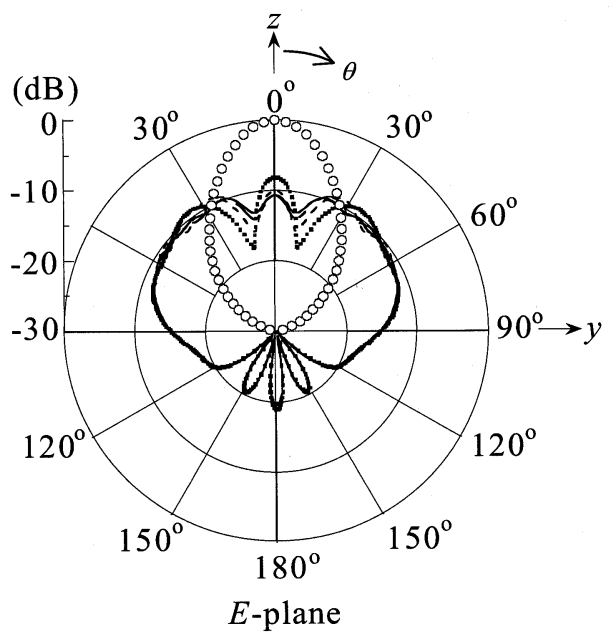

(a)

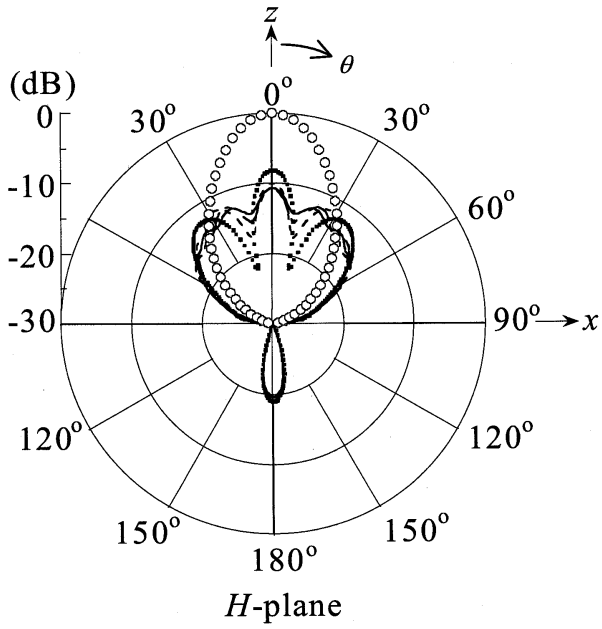

(b)

Fig. 6. Feed and terminal patterns; terminal pattern $\circ 0 \circ$, feed pattern at $L_{\mathrm{r}}=$ $50 \mathrm{~mm} \cdots$, at $L_{\mathrm{r}}=100 \mathrm{~mm}---$, at $L_{\mathrm{r}}=150 \mathrm{~mm}-.-$, at $L_{\mathrm{r}}=200 \mathrm{~mm}-$.

reference data using only the conventional FDTD analysis. For the calculation of the directivity as a function of rod length $L_{\text {rod }}$, a virtual closed surface [20] containing a plane at $z=L_{\text {rod }}$ is placed in the computational region. The virtual closed surface is plotted by broken lines in Fig. 2. The $x-z$ and $y-z$ planes of the virtual closed surface, which is regarded as Kirchhoff-Huygens' plane, are extended to the $+z$ direction with an increase in $L_{\text {rod }}$. As mentioned in Section II-A, the dimension toward the $z$ direction in the computational region is chosen to be $300 \Delta z\left(L_{z}=300 \mathrm{~mm}\right)$ due to the limited computer memory. This allows the analysis of the rod antenna with an $L_{\text {rod }}$ up to $260 \mathrm{~mm}$. The directivity calculated from the total field based on the Kirchhoff-Huygens' plane corresponds to that of an antenna with a rod length $L_{\mathrm{rod}}$, under the condition that the reflection from the free end is neglected.

According to the discontinuity-radiation concept, the radiation pattern of the dielectric rod antenna is evaluated by superposing the terminal and feed patterns generated from the guided and unguided waves. Once the feed and terminal patterns are obtained using the two waves, we do not need to repeatedly use the 


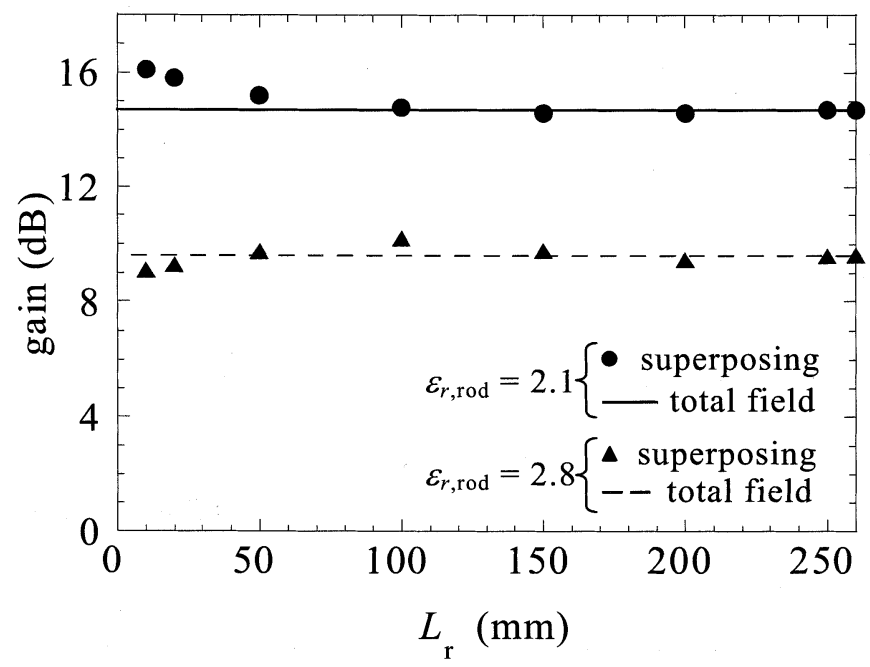

Fig. 7. Determination of sufficient length $L_{\mathrm{r}}$ (gain at $L_{\mathrm{rod}}=260 \mathrm{~mm}$ ).

FDTD method. Superposing the two patterns enables us to evaluate the radiation characteristics of a longer rod antenna without increasing the CPU time and memories.

We should recall that the leaky wave travels along the rod over an appreciable distance, as shown in Fig. 5(b). This means that the feed pattern generated by the unguided wave cannot be evaluated at the feed plane $(z=0)$, but should be evaluated at a distance where the leaky wave along the rod is negligible. To show the effects of the leaky wave, we calculate the feed patterns at various distances $L_{\mathrm{r}}$ 's. Fig. 6 shows the variation of the feed pattern for $\epsilon_{r, \text { rod }}=2.1$. The feed pattern is normalized to the maximum value of the terminal pattern illustrated for reference. It is found that the feed pattern almost converges at $L_{\mathrm{r}}=150 \mathrm{~mm}$.

As a result, the shape of the feed pattern becomes almost invariable with an increase in $L_{\text {rod }}$ provided $L_{\text {rod }}$ is more than $L_{\mathrm{r}}$. The shape of the terminal pattern is also independent of the rod length. On the other hand, the phase relation between the feed and terminal patterns changes, since the phase of the effective source at the free end delays as $L_{\mathrm{rod}}$ is increased. Therefore, the total radiation pattern $D_{\text {total }}(\theta)$ of the dielectric rod antenna with $L_{\text {rod }}$ is obtained by superposing the feed pattern $D_{\text {feed }}(\theta)$ and the terminal pattern $D_{\text {terminal }}(\theta)$ with the phase difference between the two effective sources, i.e.

$D_{\text {total }}(\theta)=D_{\text {feed }}(\theta)+D_{\text {terminal }}(\theta) \exp \left[-j\left(\beta_{0}-k_{0} \cos \theta\right) L_{\text {rod }}\right]$

where $k_{0}$ is a wavenumber in free space. Although not illustrated, good agreement is found to exist between the superposed pattern and the pattern calculated using the total fields. In other words, superposing the feed and terminal patterns forms the radiation pattern of the dielectric rod antenna.

Superposing the two patterns allows us to calculate the gain for an arbitrarily long rod antenna. Before calculating the gain, we again check the sufficient length $L_{\mathrm{r}}$. Fig. 7 shows the gain for $L_{\mathrm{rod}}=260 \mathrm{~mm}$ as a function of $L_{\mathrm{r}}$. The results are presented for both $\epsilon_{r, \text { rod }}=2.1$ and 2.8. It is found that the gain almost converges, provided $L_{\mathrm{r}}$ is more than $150 \mathrm{~mm}$.

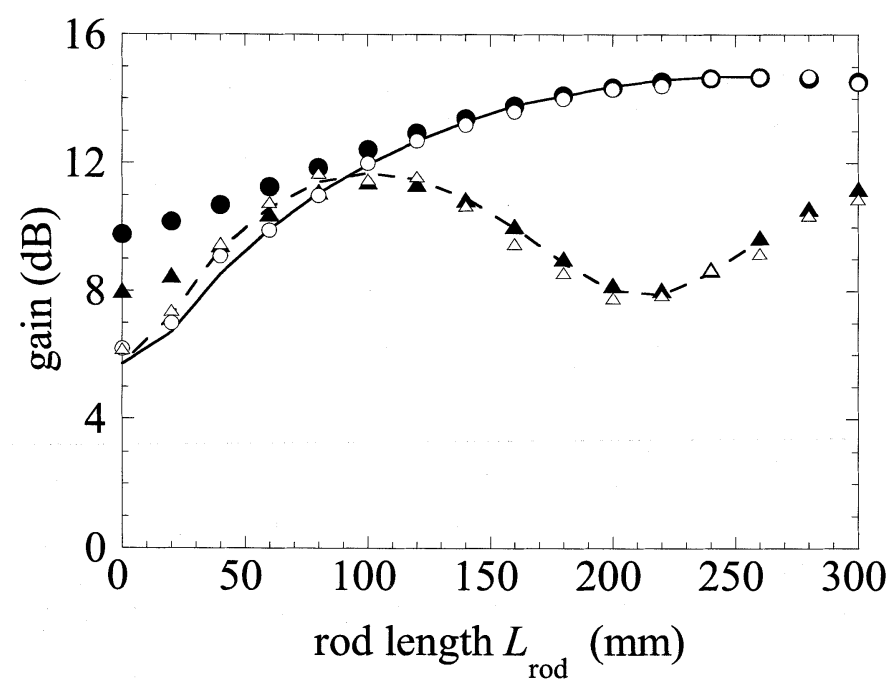

$\varepsilon_{r, \text { rod }}=2.1\left\{\begin{array}{l}\bullet \text { superposing } \\ \frac{\text { total field }}{\sigma} \text { experimental }\end{array} \varepsilon_{r, \text { rod }}=2.8\left\{\begin{array}{l}\Delta \text { superposing } \\ -- \text { total field } \\ \Delta \text { experimental }\end{array}\right.\right.$

Fig. 8. Gain as a function of rod length.

We now calculate the gain as a function of $L_{\text {rod }}$. Fig. 8 shows the gain obtained by superposing the two patterns (solid circles), together with the data obtained from the total fields (solid line). There is essentially no difference between the results obtained from the superposition and the total fields over a range of $L_{\text {rod }}$ from $120 \mathrm{~mm}$ to $260 \mathrm{~mm}$. The gain for $\epsilon_{r, \text { rod }}=2.1$ reaches a maximum value of $14.7 \mathrm{~dB}$ at $L_{\mathrm{rod}}=260 \mathrm{~mm}$. Note that the information needed for the superposition is calculated at $L_{\mathrm{r}}=$ $150 \mathrm{~mm}$. Therefore, as $L_{\mathrm{rod}}$ is decreased from $150 \mathrm{~mm}$, the superposition data induce errors.

Fig. 8 also shows the gain for $\epsilon_{r \text {,rod }}=2.8$. It is well known that the change in $\epsilon_{r, \text { rod }}$ affects the gain characteristic and that the peak gain decreases with an increase in $\epsilon_{r, \text { rod. }}$. The decrease in the peak gain is attributed to the fact that the terminal pattern becomes wider as $\epsilon_{r, \text { rod }}$ is increased, reducing the effective source area at the free end.

It is worth mentioning that the use of superposition technique drastically reduces the CPU time and memories, as compared with the FDTD analysis, when a long rod antenna is treated. For the FDTD analysis, when the $L_{\text {rod }}$ is doubled, the required memories and calculation steps are also doubled. Although an increase in the calculation steps results in an increase in the CPU time, the rate of the CPU time increase is not linear, since we have to choose a longer duration time with an increase in $L_{\text {rod }}$ for numerical convergence. For example, we need $45 \mathrm{~min}$ and $230 \mathrm{MB}$ in the FDTD analysis for $L_{\mathrm{rod}}=150 \mathrm{~mm}$ on a Pentium 600-MHz PC. After this calculation, we can calculate any longer antenna with almost no additional CPU time using the superposition technique. In contrast, if we directly analyze the antenna with $L_{\mathrm{rod}}=260 \mathrm{~mm}$, more than 100 min are needed. Furthermore, we can not calculate a longer antenna over $L_{\text {rod }}=$ $260 \mathrm{~mm}$, owing to the limitation of memories. In summary, superposing the two patterns enables us to efficiently calculate the directivity of a long rod antenna. 


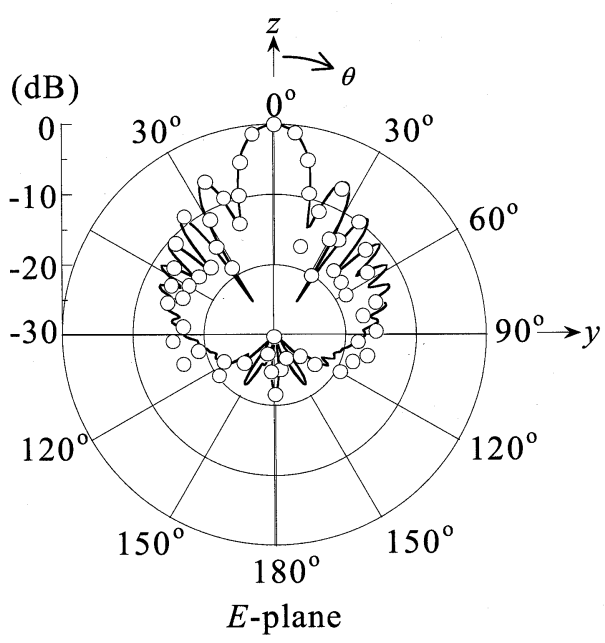

(a)

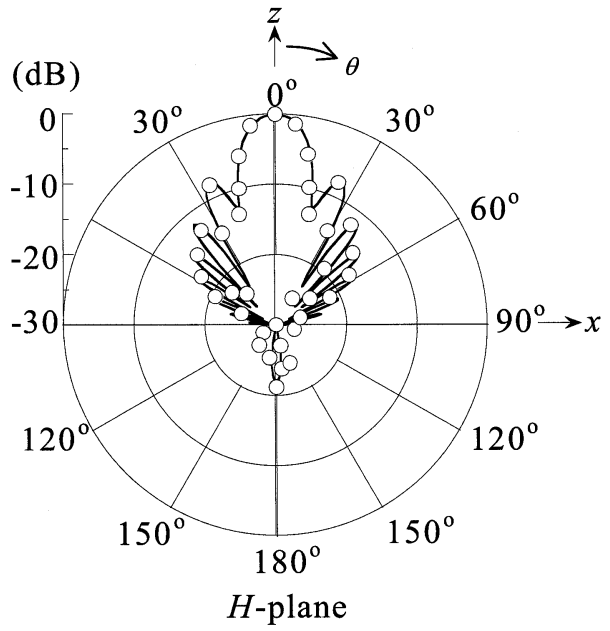

(b)

Fig. 9. Radiation pattern of a dielectric rod antenna $\left(L_{\mathrm{rod}}=260 \mathrm{~mm}\right)$; theoretical一, experimental $\circ$.

For confirming the theoretical results, we performed experiments. Recall that the free end is terminated with air in practice. The reflected wave is generated at the free end, owing to the impedance mismatch between the rod and the air region. The reflected wave causes a slight periodic variation in the gain as a function of rod length [23]. In the model of $\epsilon_{r, \text { rod }}=2.1$, the reflected power is calculated to be $0.9 \%$. To reduce the reflected power, we introduce an antireflective (AR) layer by analogy with photonics applications [24].

The configuration parameters of the AR layer for $\epsilon_{r, \text { rod }}=$ 2.1 are determined as follows [23]: The dimensions of the cross section are chosen to be the same as those of the $\operatorname{rod} a \times b$ so that the layer may not shield the unguided wave. The relative permittivity, $\epsilon_{r, \mathrm{AR}}\left(=\sqrt{\epsilon_{r, \mathrm{rod}} \epsilon_{r, \text { air }}}\right.$, where $\epsilon_{r \text {,air }}$ is the relative permittivity of air), is taken to be 1.45 . To obtain an optimum length $L_{\mathrm{AR}}$, we performed preliminary calculations using the FDTD method. When $L_{\mathrm{AR}}$ is chosen to be $11 \mathrm{~mm}$, the reflected power is reduced to $0.07 \%$.

The measured gain is also plotted in Fig. 8. The average error of the measured gain was within $0.2 \mathrm{~dB}$. Good agreement is found between the theoretical and experimental results.
Although not illustrated, we numerically and experimentally confirmed that the slight periodic variation in the gain against $L_{\text {rod }}$ was successfully suppressed by the use of the AR layer.

Fig. 9 presents the typical radiation pattern for $\epsilon_{r, \text { rod }}=2.1$ at $L_{\text {rod }}=260 \mathrm{~mm}$. Experimental data (open circles) show a good correlation with the theoretical data (solid lines) that are obtained by superposing the feed and terminal patterns in Fig. 6. The radiation pattern was measured in an anechoic chamber. Slight discrepancies between the experimental and calculated data toward the $-z$ direction are due to the existence of the feeding circuit placed behind the ground plane.

We finally make a brief comment on the total antenna efficiency defined as $\eta_{\mathrm{t}}=\eta_{\mathrm{r}} \eta_{\mathrm{d}}$, where $\eta_{\mathrm{r}}$ is the reflection efficiency deteriorated by the mismatch between the rod and the metallic waveguide, and $\eta_{\mathrm{d}}$ is the dielectric efficiency deteriorated by the electric loss tangent (the dielectric rod has so far been assumed to be lossless). Taking into account the electric loss tangent of Teflon $\left(\tan \delta=3 \times 10^{-4}\right.$ [25]), we evaluate the dielectric efficiency. As a result, $\eta_{\mathrm{d}}$ is calculated to be 0.995 for $L_{\mathrm{rod}}=$ $260 \mathrm{~mm}$, which means a negligible loss in practice. Hence, the antenna efficiency is mainly determined by $\eta_{\mathrm{r}}$. Note, that we did not employ any impedance matching technique at the feed end (see Fig. 1). Therefore, the total antenna efficiency of the present antenna is calculated to be 0.82 for $L_{\text {rod }}=260 \mathrm{~mm}$. It should be noted that the impedance matching can be realized, for example, by a technique in which a portion of the rod is inserted into the waveguide (a return loss of greater than $25 \mathrm{~dB}$, corresponding to $\eta_{\mathrm{r}} \cong 0.997$, is obtained) [26]. Consequently, a total antenna efficiency of greater than 0.99 is achieved by introducing the impedance matching technique.

\section{CONCLUSION}

We have demonstrated the discontinuity-radiation concept for an antenna system in which a dielectric rod is fed by a rectangular waveguide with a planar ground plane. The FDTD method is applied to the analysis of the total field near the rod. The imaginary-distance beam propagation method is utilized for evaluating the eigenmode in the rod.

The field near the rod is decomposed into the guided and unguided waves. Using the two waves, the feed and terminal patterns are evaluated. It is numerically demonstrated that superposing the feed and terminal patterns forms the radiation pattern of the dielectric rod antenna. The technique of superposing the two patterns has the advantage that the gain of a long rod antenna can efficiently be evaluated. The radiation characteristics calculated on the basis of the discontinuity-radiation concept agree well with the experimental results.

\section{ACKNOWLEDGMENT}

The authors would like to thank Prof. S. Kawakami of Tohoku University for his valuable suggestions regarding the mode decomposition.

\section{REFERENCES}

[1] R. E. Collin and F. J. Zucker, Antenna Theory. New York: McGrawHill, 1969, pt. 2. 
[2] J. B. Andersen, Metallic and Dielectric Antennas, Denmark: Polyteknisk Forlag, 1971.

[3] J. R. James, "Engineering approach to the design of tapered dielectric-rod and horn antennas," Radio Electron. Eng., vol. 42, no. 6, pp. $251-259,1972$

[4] F. J. Zucker, "Surface-wave antennas and surface-wave excited arrays," in Antenna Engineering Handbook, 2nd ed, R. C. Johnson and H. Jasik, Eds. New York: McGraw-Hill, 1984, ch. 12.

[5] Y. Shiau, "Dielectric rod antennas for millimeter-wave integrated circuits," IEEE Trans. Microwave Theory Tech., vol. MTT-24, pp. 869-872, Nov. 1976.

[6] T. Takano and Y. Yamada, "The relation between the structure and the characteristics of a dielectric focused horn," Trans. IECE, vol. J60-B, no. 8, pp. 395-593, 1977.

[7] S. Kobayashi, R. Mittra, and R. Lampe, "Dielectric tapered rod antennas for millimeter-wave applications," IEEE Trans. Antennas Propagat., vol. 30, pp. 54-58, Jan. 1982.

[8] C. Yao and S. E. Schwarz, "Monolithic integration of a dielectric millimeter-wave antenna and mixer diode: an embryonic millimeter-wave IC," IEEE Trans. Microwave Theory Tech., vol. MTT-30, pp. 1241-1247, Aug. 1982.

[9] R. Chatterjee, Dielectric and Dielectric-Loaded Antennas, U.K.: Research Studies Press, 1985

[10] F. Schwering and A. A. Oliner, "Millimeter-wave antennas," in Antenna Handbook, Y. T. Lo and S. W. Lee, Eds. New York: Van Nostrand Reinhold, 1988, ch. 17.

[11] M. Koshiba, Optical Waveguide Analysis. New York: McGraw-Hill, 1990 , ch. 5.

[12] C. Vassallo, "1993-1995 Optical mode solvers," Optic. Quantum Electron., vol. 29, pp. 95-114, 1997.

[13] D. Yevick and W. Bardyszewski, "Correspondence of variation finitedifference (relaxation) and imaginary-distance propagation methods for modal analysis," Opt. Lett., vol. 17, no. 5, pp. 329-330, 1992.

[14] S. Xiao, R. Vahldieck, and H. Jin, "Full-wave analysis of guided wave structure using a novel 2-D FDTD," IEEE Microwave Guided Wave Lett., vol. 2, pp. 165-167, 1992.

[15] A. Asi and L. Shafai, "Dispersion analysis of anisotropic inhomogeneous waveguides using compact 2D-FDTD," Electron. Lett., vol. 28, pp. 1451-1452, 1992.

[16] S. M. Lee, "Finite-difference vectorial-beam-propagation method using Yee's discretization scheme for modal fields," J. Opt. Soc. Amer. A, Opt. Image Sci., vol. 13, no. 7, pp. 1369-1377, 1996.

[17] J. Yamauchi, N. Morohashi, and H. Nakano, "Rib waveguide analysis by the imaginary-distance beam-propagation method based on Yee's mesh," Optic. Quantum Electron., vol. 30, pp. 397-401, 1998.

[18] T. Ando, J. Yamauchi, and H. Nakano, "Demonstration of the discontinuity-radiation concept for a dielectric rod antenna," in Proc. IEEE AP-S Int. Symp. Dig., 2000, pp. 856-859.

[19] S. T. Chu, W. P. Huang, and S. K. Chaudhuri, "Simulation and analysis of waveguide based optical integrated circuits," Comput. Phys. Coтmunicat., vol. 68, pp. 451-484, 1991.

[20] A. Taflove and S. C. Hagness, Computational Electrodynamics, The Finite-Difference Time-Domain Method, 2nd ed. Norwood, MA: Artech House, 2000.

[21] O. M. Ramahi, "The concurrent complementary operators method for FDTD mesh truncation," IEEE Trans. Antennas Propagat., vol. 46, pp. $1475-1482$, Oct. 1998

[22] A. W. Snyder and J. D. Love, Optical Waveguide Theory. London, U.K. Chapman \& Hall, 1983, ch. 24

[23] J. Yamauchi, T. Ando, and H. Nakano, "FD-TD analysis of dielectric rod antennas with an antireflective layer," in Proc. IEEE AP-S Int. Symp. Dig., 1999, pp. 602-605.

[24] J. Yamauchi, M. Mita, S. Aoki, and H. Nakano, "Analysis of antireflection coatings using the FD-TD method with the PML absorbing boundary condition," IEEE Photon. Technol. Lett., vol. 8, pp. 239-241, Feb. 1996
[25] C. A. Balanis, Advanced Engineering Electromagnetics. New York: Wiley, 1989.

[26] T. Ando, J. Yamauchi, and H. Nakano, "Rectangular dielectric-rod fed by a metallic waveguide," Proc. Inst. Elect. Eng.-Microwave Antennas Propagation, vol. 149, no. 2, pp. 92-97, 2002.

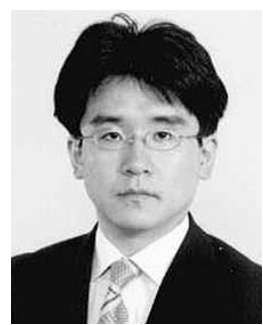

Takashi Ando (SM'99) was born in Ibaraki, Japan, on March 14, 1969. He received the B.E. and M.E. degrees from Hosei University, Tokyo, Japan, in 1991 and 1993, respectively, where he is currently working toward the Dr.E. degree.

From 1993 to 1998 , he was with TDK Corporation, Tokyo. His research interests include dielectric antennas and optical waveguides.

Mr. Ando is a Member of the Institute of Electronics, Information and Communication Engineers (IEICE) of Japan.

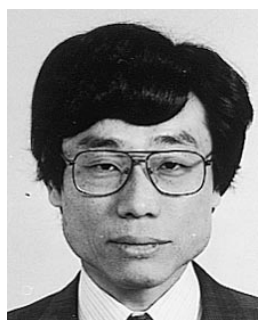

Junji Yamauchi (M'85) was born in Nagoya, Japan, on August 23, 1953. He received the B.E., M.E., and Dr.E. degrees from Hosei University, Tokyo, Japan, in 1976, 1978, and 1982, respectively.

From 1984 to 1988 , he served as a Lecturer in the Electrical Engineering Department, Tokyo Metropolitan Technical College, Tokyo, Japan. Since 1988, he has been a member of the faculty of Hosei University, where he is now a Professor of electronic informatics. His research interests include optical waveguides and circularly polarized antennas.

Dr. Yamauchi is a Member of the Optical Society of America (OSA), Washington, DC and the Institute of Electronics, Information and Communication Engineers of Japan.

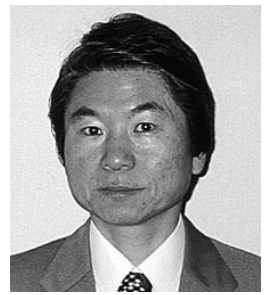

Hisamatsu Nakano (M'75-SM'87-F'92) was born in Ibaraki, Japan, on April 13, 1945. He received the B.E., M.E., and Dr.E. degrees in electrical engineering from Hosei University, Tokyo, Japan, in 1968,1970 , and 1974, respectively.

Since 1973, he has been a member of the faculty of Hosei University, where he is now a Professor of electronic informatics. From March to September 1981, he was a Visiting Associate Professor at Syracuse University, Syracuse, NY, from March to September 1986, he was a Visiting Professor at the University of Manitoba, MB, Canada, and from September 1986 to March 1987, a Visiting Professor at the University of California, Los Angeles. He has published more than 160 referred journal papers and 120 international symposium papers on antenna and relevant problems. He is the author of Helical and Spiral Antennas (New York: Research Studies Press, Wiley, 1987). He published the chapter "Antenna analysis using integral equations," in Analysis Methods of Electromagnetic Wave Problems, vol. 2 (Norwood, MA: Artech House, 1996). His research topics include numerical methods for antennas, electromagnetic wave scattering problems, and light wave problems.

Dr. Nakano received an International Scientific Exchange Award from the Natural Sciences and Engineering Research Council of Canada. In 1987, he received the Best Paper Award from the IEE 5th International Conference on Antennas and Propagation. In 1994, he received the IEEE AP-S Best Application Paper Award (H.A. Wheeler Award). He is an Associate Editor of the IEEE AntenNas and PRopagation Magazine. He was a Member of the AP-S Administrative Committee in 2002. 